Comparison of electrostatic and localized plasmon induced light enhancement in hybrid InGaN/GaN quantum wells

Jie Lin, Antonio Llopis, Arkadii Krokhin, Sergio Pereira, Ian M. Watson, and Arup Neogi'

Citation: Appl. Phys. Lett. 104, 242106 (2014); doi: 10.1063/1.4884075

View online: $\mathrm{http}: / / d x . d o i . o r g / 10.1063 / 1.4884075$

View Table of Contents: http://aip.scitation.org/toc/apl/104/24

Published by the American Institute of Physics

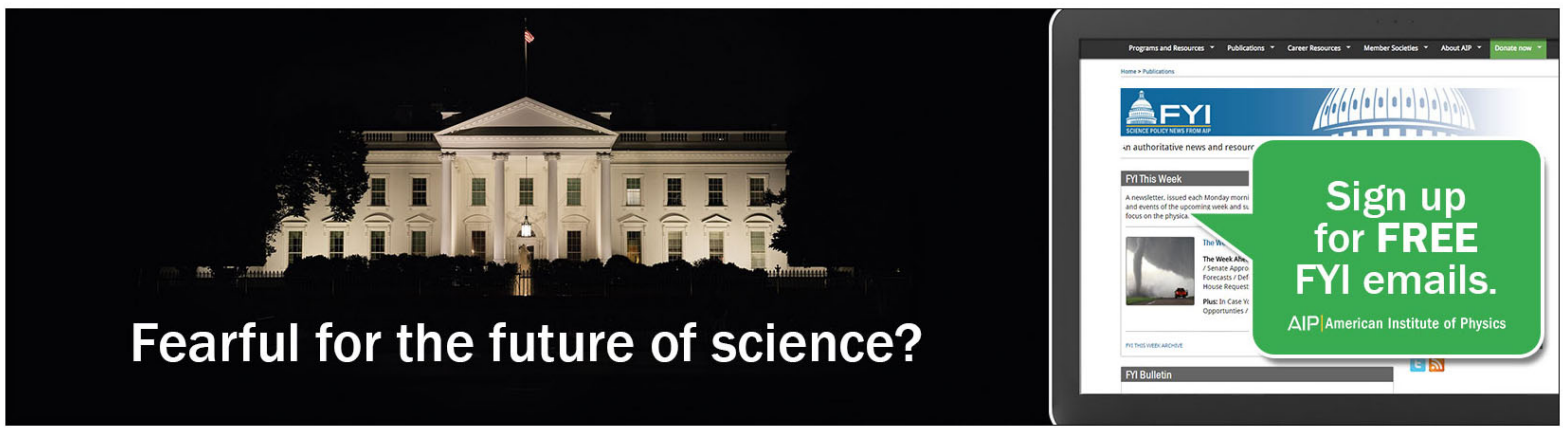




\title{
Comparison of electrostatic and localized plasmon induced light enhancement in hybrid InGaN/GaN quantum wells
}

\author{
Jie Lin, ${ }^{1}$ Antonio Llopis, ${ }^{1}$ Arkadii Krokhin, ${ }^{1}$ Sergio Pereira, ${ }^{2}$ Ian M. Watson, ${ }^{3}$ \\ and Arup Neogi, a) \\ ${ }^{1}$ Department of Physics, University of North Texas, Denton, Texas 76203, USA \\ ${ }^{2}$ CICECO, University of Aveiro, 3810193 Aveiro, Portugal \\ ${ }^{3}$ SUPA, Institute of Photonics, University of Strathclyde, Glasgow, United Kingdom
}

(Received 8 April 2014; accepted 6 June 2014; published online 18 June 2014)

\begin{abstract}
The light enhancement phenomena in InGaN/GaN multi-quantum wells (MQWs) infiltrated with metal nanoparticles (NPs) are studied using resonant and off-resonant localized plasmon interactions. The emission and recombination characteristics of carriers in InGaN/GaN MQW structures with inverted hexagonal pits (IHPs) are modified distinctly depending on the nature of their interaction with the metal NPs and with the pumping and emitted photons. It is observed that the emission intensity of light is significantly enhanced when the emission energy is off-resonant to the localized plasmon frequency of the metal nanoparticles. This results in enhanced emission from MQW due to Au nanoparticles and from IHPs due to Ag nanoparticles. At resonant-plasmon frequency of the Ag NPs, the emission from MQWs is quenched due to the re-absorption of the emitted photons, or due to the drift carriers from c-plane MQWs towards the NPs because of the Coulomb forces induced by the image charge effect. @ 2014 AIP Publishing LLC. [http://dx.doi.org/10.1063/1.4884075]
\end{abstract}

Metal-semiconductor hybrid structures are increasingly used for the enhancement of the efficiency of light emitters. In optoelectronics, InGaN is an important component for the production of high efficiency UV, blue, and green light emitting diodes (LEDs) and laser diodes. ${ }^{1,2}$ In order to enhance the commercial viability of InGaN-based components, such as white-light LEDs, the overall efficiency of the devices must be increased. ${ }^{3}$ One method of light enhancement is to increase the recombination efficiency of the system via coupling to surface plasmons, as was demonstrated by our group in various semiconductor light emitting material system. ${ }^{4-6}$ Despite the large increase in the spontaneous emission rate, however, surface plasmon mediated enhancement of light emission is limited by Joule heating and non-radiative recombination at the metal-semiconductor interface. ${ }^{7-9}$

One possible way to reduce Joule heating is to utilize localized surface plasmons (LSPs) using metal nanoparticles $(\mathrm{NPs})^{5,7}$ The presence of metal NPs on or embedded within light emitters also facilitates the tunability of the localized plasmon frequency compared to long range surface plasmon frequency, which is usually fixed in case of thin film. In case of the thin films, the dielectric constant of the metal and the semiconductor surface characterizes the LSP energy and thereby the upper limit of the photons energy of the emitter that can be resonantly coupled with the plasmons. Moreover, for monodisperse NPs, the LSP modes can have a very narrow bandwidth. In case of optical pumping, the incident laser light can thereby be used to control the presence of the plasmonic mode by tuning the frequency of the laser with respect to the LSP energy. The light enhancement from silicon based light emitters has been observed using silver ( $\mathrm{Ag}$ ) or gold (Au) nanoparticles on the surface of the emitter with an emission energy, which is significantly off-resonant to the

\footnotetext{
${ }^{\text {a) }}$ Author to whom correspondence should be addressed. Electronic mail: arup@unt.edu.
}

plasmon frequency of the metal structures. ${ }^{10}$ Recently, it has been reported that the presence of metal nanoparticles can yield significant enhancement even without any coupling of the emitted photons to the localized surface plasmon modes of metal nanoparticles embedded within the active layer of a semiconductor emitter. Electrostatic effects have been shown to produce off-resonant enhancement in the presence of metal NPs. ${ }^{11}$

In this communication, we study the light emission characteristics of an InGaN/GaN-based multiple quantum well (MQW) structure in both resonant and off-resonant conditions by embedding Au and Ag NPs within the MQW. The localized surface plasmon energy of metal nanoparticles depends on the size of the nanoparticles. The LSP energy due to metal nanoparticles is always lower than the long range surface plasmon energy of the symmetric mode at the metaldielectric interface. $\mathrm{GaN} / \mathrm{Ag}$ has a surface plasmon frequency $\sim 3.0 \mathrm{eV}$. If an InGaN/GaN quantum well emission energy is chosen to overlap the LSP energy, a strong quantum electrodynamical coupling between the quantum well and the surface plasmon can take place, with an electron hole pair in the semiconductor spontaneously recombining and producing a surface plasmon, instead of a free-space photon.

The InGaN/GaN MQWs studied here were grown on sapphire (001) substrate in a metal-organic chemical vapor deposition (MOCVD) reactor. A GaN buffer of $1 \mu \mathrm{m}$ thickness was grown on the substrate at set-point temperature $1140{ }^{\circ} \mathrm{C} .14$ periods of $\mathrm{InGaN} / \mathrm{GaN}$ MQWs were then grown at $860^{\circ} \mathrm{C}$, and then capped with GaN layer (Fig. 1(a)). Under these growth conditions, the threading dislocation (TD) density is about $2-3 \times 10^{9} \mathrm{~cm}^{-2} \cdot{ }^{12}$ During growth, strain relaxation in the alternating $\mathrm{InGaN}$ and $\mathrm{GaN}$ layers leads to the formation of an inverted hexagonal pit (IHP) centered on the TD. Normally, the TDs should act as non-radiative recombination centers; however, it has been known for some time now that the TDs within IHPs do not act as such. Instead they just reduce the active area of the quantum wells. ${ }^{13-16}$ It 

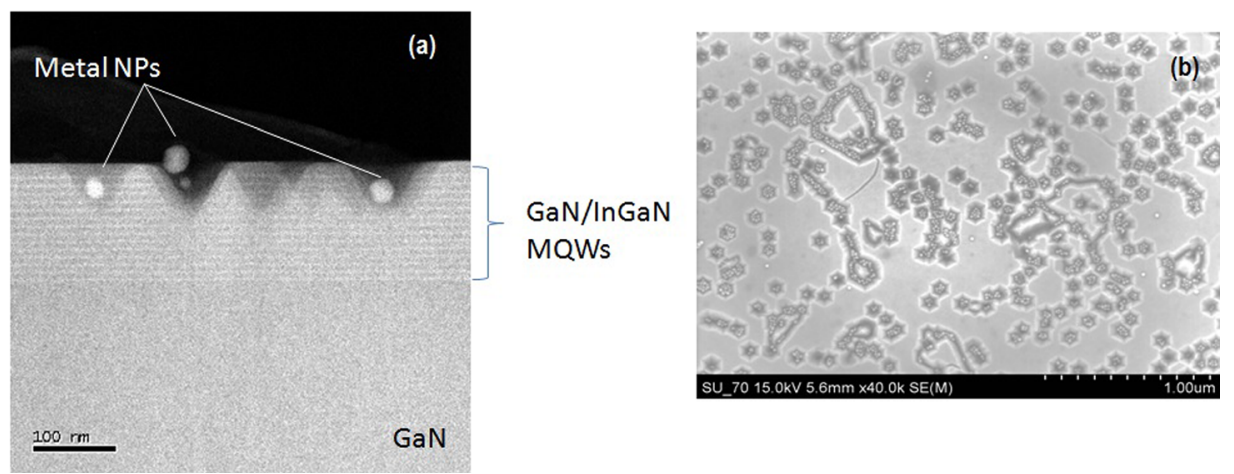

FIG. 1. Transmission electron micro scopic image and (b) scanning electron microscope image of metal nanopar ticle embedded in the IHPs or $\mathrm{V}$ pits of GaN/InGaN MQWs. was later shown by Hangleiter et al. ${ }^{14}$ that the quantum wells are significantly narrower in dimension within the pits. The narrower quantum wells result in an increase in the bandgap within the pits and results in the formation of a potential barrier that effectively shields carriers from the non-radiative recombination center at the center of the IHP. ${ }^{2,15}$ The IHPs were filled with $\mathrm{Au}$ or Ag nanoparticles as shown in Figure 1. The IHPS provide a means by which to place the NPs deep within the MQW without damaging the structure of the quantum wells. This close proximity of the localized plasmons to the excitons increases not only the plasmonic interaction but also enhances the Coulomb interaction due to image charges. ${ }^{11}$

The diameters of the implanted NPs vary from 15 to $20 \mathrm{~nm}$. The LSP energy of the nanoparticles depends on the actual environment experienced by the Au or Ag NPs within the IHPs. Due to the variation in the number of nanoparticles in the pit and the extent of metal surface surrounded by dielectric and air, the LSP energy spread can be fairly broad. The LSP energy for Au nanoparticles embedded within the IHPs has been estimated from transmission measurements using a femtosecond continuum laser generated a white light spectrum. The absorption range is observed to be significantly lower than the bandedge of the multiple quantum well and therefore does not contribute to any plasmonic interactions. The pump laser (at $325 \mathrm{~nm}$ ) and the photoluminescence (PL) from the MQWs do not excite or interact with localized plasmon due to $\mathrm{Au}$ NPs observed at $2.0 \mathrm{eV}$. However, the LSP energy of the Ag NPs cannot be directly estimated as it lies close to the absorption bandedge of the InGaN/GaN MQWs. The range of LSP for Ag nanoparticles embedded within the IHPs has been simulated using localized Mie theory. For a single Ag particle, the SP energy varied from $3.1 \mathrm{eV}$ to $3.8 \mathrm{eV}$ with varied particle size. ${ }^{17}$ In the IHPs, the primary factors affecting the LSP energy are the presence of other NPs and the ratio of surface in close proximity to GaN vs close proximity to air. While the actual plasmon energy in this system is quite complicated to solve for, we have estimated it by modeling the surrounding material as an effective dielectric medium. We then take the index of refraction to be that of a material comprised a given ratio of $\mathrm{GaN}$ to air. The LSP energy for the structure is shown in Figure 2 for Ag NPs surrounded by $50 \%$ and $75 \%$ air. The distribution of the nanoparticles within the pits is heterogeneous as shown in Figure 1.

The optical characterization was performed using both continuous-wave (CW) PL and time-resolved PL (TRPL) measurements. For the $\mathrm{CW}$ measurements, a near-field scanning optical micro-spectrometer (JASCO NFS-330) was used in illumination-collection mode to measure the nearfield photoluminescence excited with a $325 \mathrm{~nm} \mathrm{HeCd}$ laser. As both the excitation and emission were in the near$\mathrm{UV} / \mathrm{Vis}$, a $120 \mathrm{~nm} \mathrm{UV}$ probe was selected for these measurements. For the TRPL measurements, the samples were excited by a femtosecond Ti:sapphire laser (Spectra-Physics Mai Tai with a $80 \mathrm{MHz}$ rep. rate) at $712 \mathrm{~nm}$ doubled to $356 \mathrm{~nm}$. The TRPL was measured using a Streak Camera (Hamamatsu Streak Scope C4334) with a temporal resolution of 15 ps.

Figure 3 shows the near-field PL spectra of the MQW structures. It has been observed that, due to the IHPs, the well width of InGaN quantum wells within the IHPs can narrow down from $2.5 \mathrm{~nm}$ to $1.2 \mathrm{~nm}$, while the barrier width or the GaN thickness can reduce from 7.5 to $1.1 \mathrm{~nm} .^{14}$ This sharp drop in the thickness of the GaN spacer between the quantum wells leads to a coupling between the quantum wells in the IHP. ${ }^{15}$ The PL spectrum for a reference sample without any metal nanoparticles is shown in Figure 3(a). The narrowing of the QWs results in a distinct emission at a higher energy, which may be extracted via a Gaussian fit of the spectra. For clarity, we will refer to the emission from the MQW outside the IHPs as the c-plane emission or peak, and that from within the IHP as the IHP emission. When the structure is implanted with Au nanoparticles (Figure 3(b)), it is observed that the emission from the c-plane MQWs is

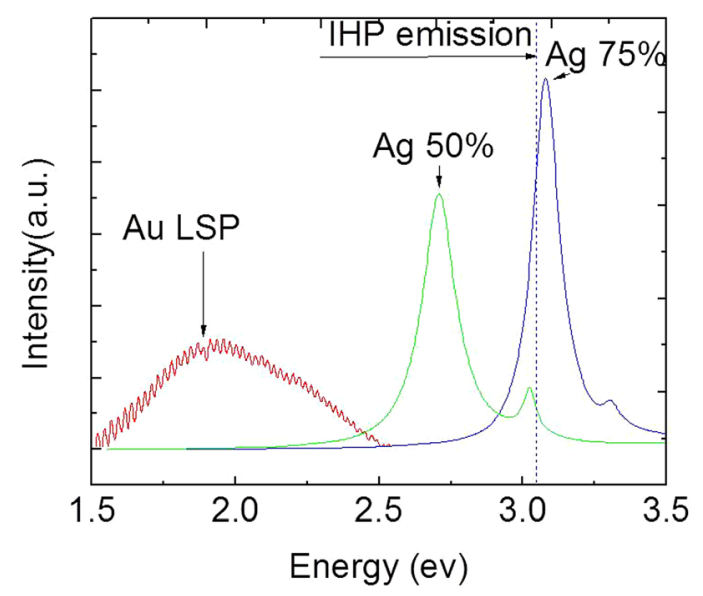

FIG. 2. Localized surface plasmon energy experimentally measured for Au nanoparticles. As the LSP energy of Ag is close to band edge of the InGaN/GaN MQWs, a simulated plot of the LSP showing the upper and lower limit of the infiltrated $\mathrm{Ag}$ nanoparticle surrounded by air within pits. 

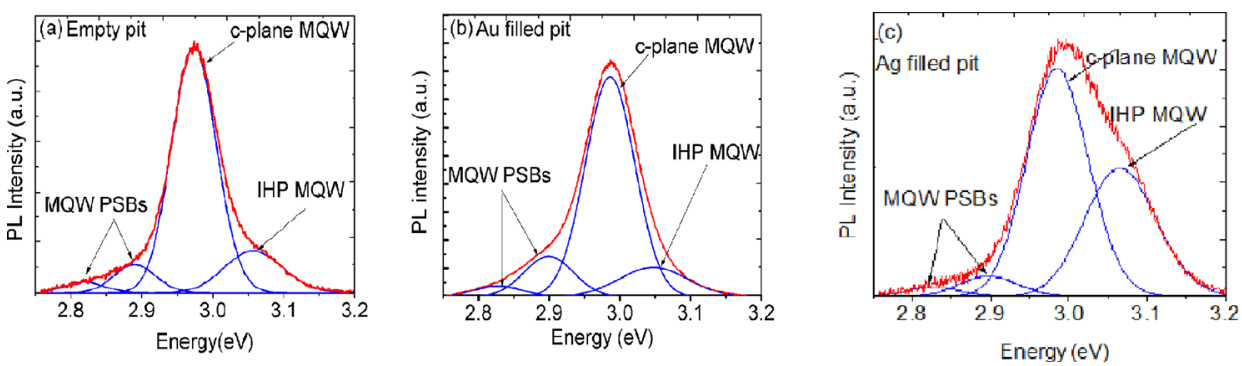

FIG. 3. Near field photoluminescence spectrum from InGaN/GaN MQWs from the vicinity of the $\mathrm{IH}$ pits showing emis sion from c plane MQWs, IHP MQWs and phonon side bands (a) reference, (b) $\mathrm{Au}$ infiltrated InGaN MQWs, (c) Ag infiltrated InGaN MQWs. significantly enhanced compared to the reference sample, and the peak emission energy is slightly blue shifted due to the image charge effect at low temperature. ${ }^{11}$ The IHP peak emission is weak as the carriers recombine mainly through the lower energy states, which is more efficient due to the accumulation of charger carriers around the metal nanoparticles.

Figure 4 shows a comparison of the far-field PL spectrum from the MQWs infiltrated with $\mathrm{Au}$ and Ag nanoparticles. At room temperature (Figure 4(a)), the emission from $\mathrm{Ag}$ infiltrated InGaN/GaN MQWs is lower than that of the reference or the Au filled structure. Even at low temperatures (Fig. 4(b)), when the non-radiative recombination rate is significantly reduced, the PL emission intensity from the $\mathrm{Ag}$ infiltrated MQWs is significantly lower than the Au infiltrated quantum wells and is only slightly brighter than the reference samples. The PL enhancement in case of Ag NPs infiltrated InGaN MQW is a three step process. The first step is the absorption of excitation photon at $\sim 3.40 \mathrm{eV}$ by the quantum well, followed by an enhancement of the light emission from the MQWs via coupling to the resonant LSP modes that subsequently couples to far-field radiation and emits the photons. ${ }^{16}$ However, a fraction of the light generated within the MQWs is absorbed and a part of the generated photons are scattered by the metal nanoparticles with plasmon frequency resonant to the emission energy of the quantum well. In case of Au NPs, as the LSP energy is detuned from the emission energy of the MQW, the reabsorption or scattering by the NP does not affect the emission process. In case of the Au infiltrated MQWs, the process of light emission is dictated carrier accumulation due to the drift and diffusion of induced carrier transport from regions away from the IHP pits to the metallic structures. ${ }^{11}$

It can also be observed from Figure 3(b) that the intensity of emission from the IHP peak is significantly enhanced compared to that of the reference sample. A comparison of the LSP energy plotted in Figures 2 and 3(c) shows that the IHP emission is slightly higher compared to the plasmon frequency. The photons emitted from the narrower QWs are not absorbed by the Ag nanoparticles. Instead the electrostatic image charge effect results in an enhancement of the high energy emission due to increased carrier density around the metal nanoparticles. The phonon replica of the c-plane MQW emission is also observed to be significantly stronger in case of resonant coupling with Ag NP induced plasmons compared to off-resonant plasmon excitation in case of $\mathrm{Au}$ NPs. The spectral curve fitting of the near-field PL emission shows that the IHP peak emission is stronger in the presence of Ag NPs compared to the reference MQWs without any nanoparticles. The LSP modes due to Ag nanoparticles couple with IHP emission energy at $3.07 \mathrm{eV}(405 \mathrm{~nm})$. This results in an enhancement of its radiative recombination rate. This resonant surface plasmon effect also was proven by the time-resolved photoluminescence results presented in Fig. 5.

Figure 5 also shows a comparison of the TRPL measurements for $\mathrm{Au}$ and $\mathrm{Ag}$ filled InGaN/GaN MQWs. It is observed that the PL lifetime of the IHP emission is shorter for $\mathrm{Ag}$ samples compared to the reference InGaN MQWs (Fig. 5(a)). This is a signature of plasmonic coupling ${ }^{6,7}$ and is caused by the enhancement of the spontaneous emission rate. The Au filled InGaN/GaN quantum wells, on the other hand, show a longer recombination time compared to the reference sample. This longer PL lifetime is due to the drift and diffusion time required for the accumulation of carriers around the metal NPs within the IHPs ${ }^{11}$ and is a signature of the image charge effect.

A comparison of the PL lifetimes as a function of energy shows that the decay time in the c-plane MQWs is significantly longer for the Au-infiltrated MQWs compared to the reference samples (Fig. 5(a)). The difference in PL lifetime is observed to be more significant for the emission from IHP QWs. This significant difference for Au filled IHP MQWs is
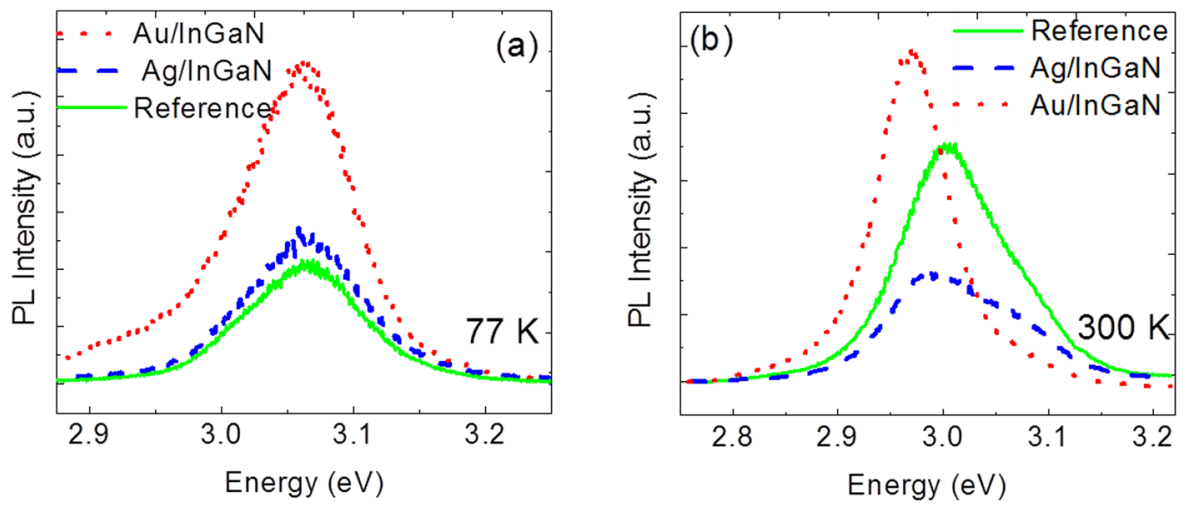

FIG. 4. Far field photoluminescence emission from InGaN/GaN MQWs samples at (a) low temperature $77 \mathrm{~K}$ (b) room temperature $300 \mathrm{~K}$. 

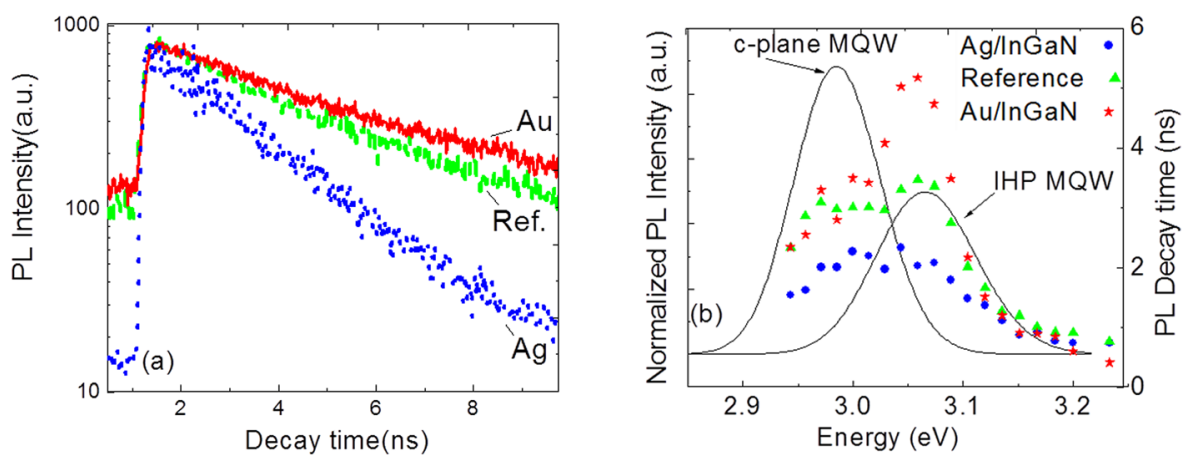

FIG. 5. Comparison of time resolved photoluminescence spectra (a) TRPL decay time behavior at $3.05 \mathrm{eV}$. (b) Energy dependence of PL recombina tion lifetime at $77 \mathrm{~K}$. due to the potential barriers caused by narrowed QWs. The narrowing in IHPs results in graded index potential well ${ }^{16}$ due to which the threading dislocation act as a sink for carriers. It hinders the carrier concentration from increasing significantly within the IHP QWs. Therefore, there is a lack of enhancement of the IHP emission due to emission due to the image charge effect in $\mathrm{Au}$ infiltrated InGaN/GaN MQW samples. In the case of the Ag-infiltrated MQWs, however, the lifetime is always shorter compared to the reference sample due to resonant surface plasmon mediated spontaneous emission in resonantly coupled $\mathrm{InGaN} / \mathrm{GaN}$ or $\mathrm{Au}$ metal infiltrated MQW systems. ${ }^{7}$ The onset of the LSP-induced spontaneous emission is significantly faster than the drift and diffusion induced electrostatic image charge effect. It leads to the dominance of plasmonic induced carrier recombination process at the resonance. IHP emission enhanced by LSP coupling in Ag infiltrated MQW is relatively more than in $\mathrm{Au}$ infiltrated MQWs due to off-resonant image charge effects.

In conclusion, we have studied the effects of resonant and off-resonant enhancement in an InGaN/GaN MQW system. It is observed that in case of resonant localized plasmon interaction, the recombination process is dominated by the modification of spontaneous emission by the plasmon modes. In the case of NPs being off-resonant with the QW, the emission is dominated by the image charge effect which is due to carrier accumulation. As the time for drift and diffusion of carriers in InGaN is significantly slower than the surface plasmon induced radiative recombination process, the electrostatic effects in this system is dominated by plasmonic effect for resonant interaction. By utilizing the fast response of resonant plasmon effects and the carrier accumulation due to the drift and diffusion process at off-resonant frequencies, the image-charge effect and localized plasmon-exciton coupling effects can be combined to enhance the efficiency and performance of metal-semiconductor photonic devices.

The authors acknowledge faculty research grants from the University of North Texas. A.N. acknowledges the support from Charn Uswachoke Development funds.

${ }^{1}$ Z. Deng, Y. Jiang, Z. Ma, W. Wang, H. Jia, J. Zhou, and H. Chen, Scientific Reports 3, 3389 (2014).

${ }^{2}$ J. Kim, Y. H. Cho, D. S. Ko, X. S. Li, J. Y. Won, E. Lee, S. H. Park, J. Y. Kim, and S. Kim, Optics Express 22, A857 (2014).

${ }^{3}$ S. Nakamura and G. Fasol, The Blue Laser Diode: GaN Based Light Emitting Diode and Lasers (Springer, Berlin, 1997).

${ }^{4}$ A. Neogi and H. Morkoç, Opt. Lett. 30, 93 (2005).

${ }^{5}$ A. K. Singh, K. G. Gryczynski, F. D. McDaniel, M. Kim, and A. Neogi, Appl. Phys. Express 3(10), 102201 (2010).

${ }^{6}$ J. Lin, A. Mohammadizia, A. Neogi, H. Morkoc, and M. Ohtsu, Appl. Phys. Lett. 97(22), 221104 (2010).

${ }^{7}$ A. Neogi, C. W. Lee, H. O. Everitt, T. Kuroda, A. Tackeuchi, and E. Yablonovitch, Phys. Rev. B 66, 153305 (2002).

${ }^{8}$ I. Gontijo, M. Boroditsky, E. Yablonovitch, S. Keller, U. K. Mishra, and S. P. DenBaars, Phys. Rev. B 60, 11564 (1999).

${ }^{9}$ B. Desiatov, I. Goykhman, and U. Levy, Nanoletters 14, 648 (2014).

${ }^{10}$ M. Pillai, K. Catchpole, T. Trupke, G. Zhang, J. Zhao, and M. Green, Appl. Phys. Lett. 88, 161102 (2006).

${ }^{11}$ A. Llopis, J. Lin, S. M. S. Pereira, T. Trindade, M. A. Martins, I. M. Watson, A. A. Krokhin, and A. Neogi, Phys. Rev. B 87, 201304(R) (2013).

${ }^{12}$ C. J. Deatcher, C. Liu, S. Pereira, M. Lada, A. G. Cullis, Y. J. Sun, O. Brandt, and I. M. Watson, Semicond. Sci. Technol. 18(4), 212218 (2003).

${ }^{13}$ G. F. Yang et al., Superlattices and Microstructures 52, 349, (2012).

${ }^{14}$ A. Hangleiter, F. Hitzel, C. Netzel, D. Fuhrmann, U. Rossow, G. Ade, and P. Hinze, Phys. Rev. Lett. 95, 127402 (2005).

${ }^{15}$ C. Netzel, H. Bremers, L. Hoffmann, D. Fuhrmann, U. Rossow, and A. Hangleiter, Phys. Rev. B 76, 155322 (2007).

${ }^{16}$ A. Llopis, J. Lin, S. Periera, and A. Neogi, IEEE J. Sel. Top. Quantum Electron. 15, 1400 (2009).

${ }^{17}$ J. A. Scholl, A. L. Koh, and J. A. Dionne, Nature 483, 421 (2012). 